\title{
Relationship between the Coenzyme A and the Carnitine Pools in Human Skeletal Muscle at Rest and after Exhaustive Exercise under Normoxic and Acutely Hypoxic Conditions
}

\author{
Raymond Friolet, * Hans Hoppeler, ${ }^{\star}$ and Stephan Krähenbühl\$ \\ * Department of Anesthesiology, and ${ }^{\ddagger}$ the Institute of Anatomy, University of Berne, and ${ }^{\S}$ Clinical Pharmacology and Toxicology, \\ University Hospital of Zurich, Switzerland
}

\begin{abstract}
Skeletal muscle CoA and carnitine metabolism were investigated in six human volunteers at rest and after exhaustive exercise under normoxic and hypoxic conditions. In comparison to the values at rest, exhaustive exercise was associated with a three- to fourfold increase in the skeletal muscle lactate, and with a twofold increase in the acetyl-CoA content, both under normoxic and hypoxic conditions. Since exercise did not significantly affect the skeletal muscle CoA radical (CoASH), total acid-soluble, or total CoA contents, the increase in the acetyl-CoA content was at the expense of short-chain acyl-CoAs different from acetyl-CoA. With exhaustive exercise, the skeletal muscle acetylcarnitine and short-chain acylcarnitine contents increased by a factor of three to four both under normoxic and hypoxic conditions. In contrast to the CoA pool, these increases were associated with a decrease in the free carnitine content, whereas the total acid-soluble and total carnitine contents were not affected by exercise. After exhaustive exercise, the skeletal muscle acetyl-CoA/CoASH ratio showed a linear correlation with the corresponding acetylcarnitine/free carnitine ratio. The plasma short-chain acylcarnitine concentration increased by a factor of two to three during exercise, and was not significantly different from the values at rest 40 min after completion of exercise. Thus, the current studies illustrate the close interaction between the $\mathrm{CoA}$ and carnitine pools in the exercising human skeletal muscle, and they underscore the important role of carnitine in maintaining the muscular CoASH content during exhaustive exercise. (J. Clin. Invest. 1994. 94:1490-1495.) Key words: acetylCoA $\cdot$ acetylcarnitine $\cdot$ lactate $\cdot$ lactate threshold $\cdot$ bicycle exercise test
\end{abstract}

\section{Introduction}

Carnitine is essential for the transport of long-chain fatty acids into the mitochondrial matrix, and therefore plays an important role in energy metabolism (1). The skeletal muscle carnitine stores are both qualitatively and quantitatively important, since

Address correspondence to Stephan Krahenbuhl, M.D., Pharm. D., Clinical Pharmacology and Toxicology, Department of Internal Medicine, University Hospital, CH-8091 Zurich.

Received for publication 23 February 1994 and in revised form 25 May 1994.

J. Clin. Invest.

(c) The American Society for Clinical Investigation, Inc. $0021-9738 / 94 / 10 / 1490 / 06 \$ 2.00$

Volume 94, October 1994, 1490-1495 fatty acids represent a major energy source and more than $95 \%$ of the total body carnitine is stored in skeletal muscle $(1,2)$. It is well established that the carnitine pool in skeletal muscle can be affected by exercise. With low- to moderate-intensity exercise (below or at the lactate threshold), either no (3) or a small increase in the short-chain acylcarnitine pool (4) has been reported. In contrast, high-intensity exercise (above the lactate threshold) causes a marked increase in the skeletal muscle shortchain acylcarnitine content with a corresponding decrease in the free carnitine content in humans $(3,5)$ and horses $(6-8)$. Direct assessment of the short-chain acylcarnitine pool showed that acetylcarnitine accounted for most of the exercise-induced increase in the skeletal muscle short-chain acylcarnitine content (5-9).

The carnitine and the CoA pools are in close relationship, mediated through the action of carnitine acyltransferases $(1,10$, 11). Since the tissue carnitine pool, in general, is considerably larger than the CoA pool (see ref. 8 and this study for skeletal muscle) and the intramitochondrially formed acylcarnitines can be exported (12), carnitine may serve as a "buffer" for the metabolically important $\operatorname{CoA}$ pool $(10,13,14)$. Evidence for this important function of carnitine has been provided by studies showing that the addition of carnitine to isolated rat heart mitochondria led to an increase in the mitochondrial [CoASH ${ }^{1}$ ]/ [acetyl-CoA] ratio and stimulated acylcarnitine formation and pyruvate oxidation $(12,15)$.

Acute hypoxia represents a metabolic stress leading to increased sympathetic activity with high plasma catecholamine concentrations (16), and to changes in fat and glucose metabolism $(16,17)$. Since strenuous endurance exercise under hypoxic conditions was associated with an increase in the skeletal muscle acylcarnitine pool (18), and since it is well established that the carnitine pool reflects different metabolic states $(2)$, it was of interest to study the skeletal muscle carnitine pool under normoxic and hypoxic conditions.

Moreover, there are only few studies where the carnitine and the CoA pools were assessed simultaneously in skeletal muscle, and the results are conflicting. In swimming rats, the skeletal muscle acetylcarnitine content increased, whereas the acetyl-CoA content decreased in comparison to the values at rest (19). In contrast, in the exercising horse, both the acetyl$\mathrm{CoA}$ and the acetylcarnitine contents of skeletal muscle increased with exercise ( 8 ). Therefore, the current studies were designed to investigate the relationship between the skeletal muscle carnitine and CoA pools in humans at rest and after exhaustive exercise under normoxic and acutely hypoxic conditions.

1. Abbreviations used in this paper: CoASH, coenzyme A radical; $K_{\text {eq }}$, equilibrium constant; RER, respiratory exchange ratio; VE, ventilation; $\dot{\mathrm{V}} \mathrm{CO}_{2}$, carbon dioxide production; $\mathrm{Vo}_{2}$, oxygen consumption; W, workload. 
Table I. Grading of the Subjects at Entry

\begin{tabular}{|c|c|c|}
\hline & Normoxia & Hypoxia \\
\hline Maximal heart rate (beats/min) & $187 \pm 8$ & $178 \pm 10$ \\
\hline \multicolumn{2}{|c|}{ Maximal blood lactate concentration } & $11.2 \pm 0.8$ \\
\hline Maximal $\dot{\mathrm{VO}}_{2}(\mathrm{ml} / \mathrm{kg} / \mathrm{min})$ & $54.6 \pm 6.1$ & $37.3 \pm 2.2 *$ \\
\hline Maximal RER & $1.14 \pm 0.13$ & $1.26 \pm 0.09$ \\
\hline Maximal workload $(W)$ & $355 \pm 42$ & $272 \pm 28^{*}$ \\
\hline \multicolumn{3}{|l|}{ Workload at lactate threshold } \\
\hline Absolute $(W)$ & $225 \pm 44$ & $135 \pm 231 *$ \\
\hline Relative (\% maximal) & $63 \pm 7$ & $50 \pm 6 *$ \\
\hline \multicolumn{3}{|l|}{ Selected workload } \\
\hline Absolute $(W)$ & $285 \pm 32$ & $211 \pm 20 *$ \\
\hline Relative (\% maximal) & $80 \pm 2$ & $78 \pm 3$ \\
\hline
\end{tabular}

A bicycle ergometer test was performed to exhaustion with increasing workloads (30 W every $3 \mathrm{~min}$ ) under normoxic and hypoxic conditions. The heart rate, blood lactate concentrations, oxygen consumption $\left(\mathrm{V}_{2}\right)$, respiratory exchange ratio (RER), and the workload at the lactate threshold were determined as described in Methods. The initial grading allowed the selection of a workload above the lactate threshold for the final exercise tests. Data are given as mean \pm SD. $P<0.05$ hypoxia vs normoxia.

\section{Methods}

Subjects. Six healthy male subjects were enrolled in the study. The subjects were all lowlanders on no actual medication. Their mean body weight was $80 \pm 8 \mathrm{~kg}$ and their mean age $31 \pm 6$ yrs. All subjects performed exercise on a regular basis but none was involved in competitive athleticism. Informed consent was obtained from each subject participating. The study protocol was approved by the Ethical Committee of the Medical School at the University of Berne.

Initial grading. All subjects underwent two bicycle exercise tests, in random order, to characterize the individual metabolic response to increasing work load. One test was accomplished under normoxic conditions ( $21 \%$ oxygen and $79 \%$ nitrogen) and the other under hypoxic conditions ( $13.8 \%$ oxygen and $86.2 \%$ nitrogen). The gas mixtures were provided by a 250 -liter mixing chamber. The oxygen concentration of the gas mixture leaving the mixing chamber was continuously monitored with a paramagnetic oxygen analyzer (Servomex 570; Process Control Instrumentation, Esslingen, Switzerland) and kept constant by adjusting the inlet of oxygen and nitrogen. Exercise testing was performed until exhaustion on an electrically braked bicycle ergometer (Ergoline Ergometrics 800S; Leuenberger Medizin Technik AG, Glattbrugg, Switzerland). Before starting, the subjects rested on the bicycle for $10 \mathrm{~min}$, breathing the selected gas mixture. Then, they performed a warmup period consisting of loadless pedalling for $5 \mathrm{~min}$. The grading exercise tests were started at $100 \mathrm{~W}$ (normoxia) or at $70 \mathrm{~W}$ (hypoxia) with increments of $30 \mathrm{~W}$ per $3 \mathrm{~min}$. The heart rate, ventilation (VE), oxygen consumption $\left(\dot{\mathrm{V}}_{2}\right)$, carbon dioxide production $\left(\mathrm{V}_{\mathrm{CO}_{2}}\right)$ and the respiratory exchange ratio (RER, calculated as $\dot{\mathrm{V}} \mathrm{CO}_{2} / \dot{\mathrm{V}}_{2}$ ) were determined at rest (warmup period) and were monitored continuously during exercise by standard techniques of open circuit spirometry (EOS Sprint; Jaeger GmbH, Würzgurg, Germany). The oxygen and carbon dioxide analyzers were calibrated before every exercise test with standard gases of known concentration. The blood lactate concentration was determined in capillary blood obtained from the earlobe every $3 \mathrm{~min}$ before increasing the workload.

The lactate threshold was determined using the method proposed by Spurway (20) as the workload where the blood lactate concentration reaches $2 \mathrm{mmol} / \mathrm{liter}$. For the final exercise tests in normoxia or hypoxia, a workload of $\sim 80 \%$ of the maximal workload was selected. This workload was higher than the lactate threshold for every subject both under normoxic and hypoxic conditions (Table I).
Final exercise testing. The final exercise tests were performed 7$14 \mathrm{~d}$ after the initial grading. An intravenous catheter was placed in an antecubital vein of the left arm and kept open by flushing with heparinized saline. The subjects started to breathe the appropriate gas mixture in supine position. $10 \mathrm{~min}$ later, a needle biopsy was performed at rest in the right vastus lateralis muscle using a Bergström needle (21). The thigh was wrapped with a nonconstricting elastic bandage and the exercise test was started at the selected workload after a warmup phase of $5 \mathrm{~min}$ on the bicycle as described in the preceding section and was performed until exhaustion. At the completion of the exercise test, subjects returned quickly into the supine position still breathing the selected gas mixture and a second muscle biopsy was performed as described above in the left vastus lateralis muscle. Biopsies were taken within 90 $s$ after completion of exercise.

Blood was withdrawn during the warmup period, every 5 min during exercise and 20, 40, and $60 \mathrm{~min}$ after completion of the exercise test for the determination of the lactate, free carnitine, and acylcarnitine concentrations. The heart rate, VE, $\dot{\mathrm{VO}}_{2}, \dot{\mathrm{V}} \mathrm{CO}_{2}$, and RER were determined continuously during the warmup period and during the exercise test. In order to validate the open circuit spirometry, samples of expired air were also collected in Douglas bags during the exercise test and analyzed according to standard techniques (22). The results of these determinations agreed well with those obtained by open circuit spirometry, which will be reported in Results.

Sample preparation. For the determination of the blood lactate concentration, capillary blood samples were withdrawn from the earlobe and analyzed immediately. For the determination of the free and acylcarnitine concentrations, 5-ml blood samples were withdrawn in heparinized tubes and stored at $5^{\circ} \mathrm{C}$ until completion of the exercise test. Then, they were centrifuged at $600 \mathrm{~g}$ for $5 \mathrm{~min}$ and the plasma was removed and frozen at $-20^{\circ} \mathrm{C}$ until analysis. Muscle samples $(60-150 \mathrm{mg}$ ) were frozen immediately in liquid nitrogen and stored at $-170^{\circ}$ until analysis.

Assay methods. Free, total acid-soluble, and long-chain acylcarnitine concentrations in plasma and skeletal muscle were determined using the radioenzymatic assay originally described by Cederblad (23) with the modifications described by Brass and Hoppel (2). Plasma and muscle samples were prepared in perchloric acid and centrifuged for $2 \mathrm{~min}$ at $10,000 \mathrm{~g}$. In the supernatant, the free and the total acid-soluble carnitine concentrations were determined. The short-chain acylcarnitine concentration was calculated as the difference between the total acid-soluble and the free carnitine concentrations. In the pellet, the long-chain acylcarnitine concentration was determined. The total carnitine concentration was calculated as the sum of the total acid-soluble and the longchain acylcarnitine concentrations. The acetylcarnitine concentration was determined in the neutralized supernatant of the skeletal muscle perchloric acid preparation according to Cederblad (24).

The contents of CoASH, acetyl-CoA, total acid-soluble CoA, and long-chain acyl-CoA in skeletal muscle were determined using the radioenzymatic method originally described by Pande (25) and modified by Cederblad (24). CoASH, acetyl-CoA, and total acid-soluble CoA concentrations were determined in the same supernatants of the skeletal muscle perchloric acid preparations as used for the determination of the corresponding carnitines. CoASH and acetyl-CoA concentrations were determined at the day of the preparation of the skeletal muscle samples. The total acid-soluble CoA concentrations were determined after alkaline hydrolysis of the perchloric acid supernatants, and the short-chain acyl-CoA concentrations were calculated as the difference between the total acid-soluble CoA and the CoASH concentrations. The long-chain acyl-CoA concentrations were determined in the same pellet as used for the determination of the long-chain acylcarnitine concentrations. The total CoA concentration was calculated by addition of the total acidsoluble and the long-chain acyl-CoA concentrations. In order to validate the radioenzymatic assays, some samples were also analyzed by highperformance liquid chromatography, as described previously (26). The differences between the two methods were typically less than $10 \%$. The values reported in Results were obtained by the radioenzymatic method described above. The exact concentrations in the CoASH and acetyl-CoA standard solutions were determined spectrophotometrically $(27,28)$. 


\begin{tabular}{|c|c|c|c|c|c|}
\hline \multirow[b]{2}{*}{ Variable } & \multirow[b]{2}{*}{ Preexercise } & \multicolumn{3}{|c|}{ Exercise } & \multirow[b]{2}{*}{ Recovery } \\
\hline & & $10 \min$ & $20 \min$ & Exhaustion & \\
\hline \multicolumn{6}{|l|}{ Normoxia } \\
\hline Heart rate (beats/min) & $99 \pm 12$ & $161 \pm 11^{*}$ & $170 \pm 11^{*}$ & $176 \pm 9 *$ & $\mathrm{ND}^{8}$ \\
\hline$\dot{\mathrm{V}}_{2}(\mathrm{ml} / \mathrm{min} / \mathrm{kg})$ & $11.2 \pm 1.7$ & $43.7 \pm 7.6^{*}$ & $46.8 \pm 6.9 *$ & $47.7 \pm 6.7 *$ & ND \\
\hline RER & $0.90 \pm 0.07$ & $1.01 \pm 0.03 *$ & $0.98 \pm 0.05 *$ & $1.02 \pm 0.03 *$ & ND \\
\hline VE (liters/min) & $26.2 \pm 4.0$ & $94.5 \pm 9.8^{*}$ & $107 \pm 9 *$ & $109 \pm 9 *$ & ND \\
\hline Blood lactate $(m M)$ & $1.52 \pm 0.45$ & $5.33 \pm 1.75^{*}$ & $6.24 \pm 1.86^{*}$ & $6.54 \pm 2.14 *$ & $1.64 \pm 0.40$ \\
\hline \multicolumn{6}{|l|}{ Plasma carnitine $(\mu M)$} \\
\hline Free carnitine & $43.1 \pm 12.0$ & $42.3 \pm 11.6$ & $39.0 \pm 11.9$ & $38.3 \pm 12.8$ & $39.8 \pm 9.0$ \\
\hline Short-chain acylcarnitine & $2.8 \pm 1.7$ & $4.5 \pm 2.3$ & $7.4 \pm 4.6^{*}$ & $9.8 \pm 5.6^{*}$ & $4.6 \pm 2.4$ \\
\hline Long-chain acylcarnitine & $4.8 \pm 0.3$ & $4.9 \pm 0.8$ & $5.0 \pm 0.4$ & $5.1 \pm 0.8$ & $4.7 \pm 0.4$ \\
\hline \multicolumn{6}{|l|}{ Hypoxia } \\
\hline Heart rate (beats/min) & $111 \pm 22$ & $166 \pm 8^{*}$ & $172 \pm 7 *$ & $173 \pm 7 *$ & $\mathrm{ND}^{8}$ \\
\hline$\dot{\mathrm{VO}}_{2}(\mathrm{~m} / \mathrm{min} / \mathrm{kg})$ & $11.0 \pm 1.7$ & $33.0 \pm 3.9^{* \pm}$ & $33.5 \pm 3.7^{* \pm}$ & $33.2 \pm 3.9^{* \ddagger}$ & ND \\
\hline RER & $0.92 \pm 0.07$ & $1.11 \pm 0.06^{* \neq}$ & $1.07 \pm 0.05^{* \ddagger}$ & $1.09 \pm 0.06^{* \ddagger}$ & ND \\
\hline VE (liter/min) & $27.2 \pm 4.3$ & $101 \pm 11^{*}$ & $113 \pm 7 *$ & $114 \pm 9 *$ & ND \\
\hline Blood lactate $(m M)$ & $1.22 \pm 0.40$ & $6.95 \pm 1.29^{* \neq}$ & $9.15 \pm 1.37^{* \neq}$ & $9.22 \pm 1.41^{* \pm}$ & $1.92 \pm 0.33$ \\
\hline \multicolumn{6}{|l|}{ Plasma carnitine $(\mu M)$} \\
\hline Free carnitine & $35.8 \pm 6.2$ & $33.9 \pm 3.8$ & $33.8 \pm 6.7$ & $32.7 \pm 4.7$ & $35.7 \pm 4.2$ \\
\hline Short-chain acylcarnitine & $5.2 \pm 4.5$ & $9.1 \pm 3.9 *$ & $8.9 \pm 2.7^{*}$ & $9.9 \pm 2.0^{*}$ & $6.4 \pm 2.6$ \\
\hline Long-chain acylcarnitine & $4.6 \pm 1.2$ & $5.0 \pm 1.3$ & $5.2 \pm 1.7$ & $5.2 \pm 1.7$ & $3.9 \pm 0.7$ \\
\hline
\end{tabular}

The subjects $(n=6)$ performed bicycle exercise to exhaustion on the selected workload (see Table I) under normoxic and hypoxic conditions. Preexercise values were obtained in the warmup phase prior to exercise, and recovery values $\mathbf{4 0}$ min after completion of exercise. Data are given as mean \pm SD. $\quad{ }^{*} P<0.05$ exercise or recovery vs preexercise. ${ }^{\ddagger} P<0.05$ hypoxia vs normoxia. ${ }^{8} \mathrm{ND}=$ not determined.

The lactate concentration in the skeletal muscle was determined in the neutralized supernatants of the perchloric acid preparations using a lactate dehydrogenase kit from Sigma Chemical Co. (kit 826-A; Buchs, Switzerland). The lactate concentrations in blood were determined enzymatically (ESAT 6661; Eppendorf, Hamburg, Germany).

Skeletal muscle noncollagen protein concentrations were determined according to Lowry (29) with BSA as a standard after alkaline hydrolysis of collagen (30).

Skeletal muscle noncollagen protein concentrations, carnitine concentrations in plasma and skeletal muscle, and $\mathrm{CoA}$ and lactate concentrations in skeletal muscle were determined in duplicate with the average of the two values reported in Results.

Statistical analysis. Values are reported as the mean \pm 1 SD. The subjects served as their own control, by comparison of the values at rest with the values during and immediately after exercise, and during recovery. The data were analyzed by a two-way analysis of variance with repeated measures (31). In the case of significant $F$-values, differences between the values at rest and during exercise or recovery were analyzed further by Student's $t$ test for paired data with Bonferroni correction. Differences between normoxic and hypoxic conditions were analyzed by Student's $t$ test for unpaired data.

\section{Results}

The current studies were designed to assess the relationship between the CoA and the carnitine pools in human skeletal muscle at rest and after exhaustive exercise under normoxic and hypoxic conditions.

At entry, the six enrolled subjects were graded using a protocol with stepwise increase of the workload under both normoxic and acutely hypoxic conditions equivalent to an altitude of 4,000 $\mathrm{m}$. As shown in Table $\mathrm{I}$, hypoxia did not significantly affect the maximal heart rate, maximal blood lactate concentration, or maximal RER, whereas maximal $\dot{\mathrm{V}}_{2}$, maximal workload, and lactate threshold were all reduced. Based on the initial grading, a workload between the lactate threshold and the maximal workload was selected for the final studies $(78-80 \%$ of maximal $\dot{\mathrm{VO}}_{2}$ ).

At the selected workload, the subjects were asked to exercise until exhaustion. The period of time during which the probands exercised was $27.5 \pm 2.7 \mathrm{~min}$ under normoxic and $21.7 \pm 2.6 \mathrm{~min}$ under hypoxic conditions $(P<0.05)$. As shown in Table II, the heart rate, $\dot{\mathrm{VO}}_{2}, \mathrm{VE}, \mathrm{RER}$, and blood lactate concentrations all increased with exercise, both under normoxic and hypoxic conditions. In comparison to normoxia, the increase in $\mathrm{Vo}_{2}$ was less pronounced and the increases in the RER and in the blood lactate concentrations were more accentuated under hypoxic conditions. In agreement with a previous study (3), exercise above the lactate threshold was associated with an increase in the plasma short-chain acylcarnitine concentration without affecting the plasma free or long-chain acylcarnitine concentrations. $40 \mathrm{~min}$ after completion of exercise, the blood lactate and the plasma short-chain acylcarnitine concentrations were not significantly different from the corresponding preexercise values.

Skeletal muscle biopsies were performed at rest and at the time point of exhaustion. The skeletal muscle noncollagen content was (rest vs. exhaustion) $150 \pm 19$ vs. $143 \pm 24 \mathrm{mg} / \mathrm{g}$ under normoxic conditions, and $169 \pm 32$ vs. $161 \pm 26 \mathrm{mg} / \mathrm{g}$ under hypoxic conditions. Since the interpretation of the skeletal muscle metabolite contents was not different when expressed per mg noncollagen protein or per $\mathrm{kg}$ wet weight, the data will be presented per $\mathrm{kg}$ wet weight.

The skeletal muscle lactate concentrations increased from 
Table III. CoA Content in Skeletal Muscle

\begin{tabular}{lrllll}
\hline & \multicolumn{2}{c}{ Normoxia } & & \multicolumn{2}{c}{ Hypoxia } \\
\cline { 2 - 3 } \cline { 5 - 6 } & Preexercise & Exhaustion & & Preexercise & Exhaustion \\
\hline CoASH & $12.6 \pm 5.1$ & $14.2 \pm 4.5$ & & $14.7 \pm 4.2$ & $14.2 \pm 3.1$ \\
Acetyl-CoA & $5.7 \pm 4.2$ & $11.1 \pm 5.0^{*}$ & & $6.7 \pm 4.4$ & $11.7 \pm 4.4 *$ \\
Short-chain acyl-CoA & $16.7 \pm 3.8$ & $14.0 \pm 2.9$ & & $16.2 \pm 2.8$ & $16.9 \pm 5.7$ \\
Total acid-soluble CoA & $29.3 \pm 4.8$ & $28.3 \pm 5.0$ & & $31.0 \pm 4.5$ & $31.1 \pm 6.0$ \\
Long-chain acyl-CoA & $1.8 \pm 2.0$ & $1.5 \pm 0.84$ & & $1.9 \pm 1.2$ & $2.0 \pm 1.3$ \\
Total CoA & $31.1 \pm 6.3$ & $29.8 \pm 5.1$ & & $32.9 \pm 4.8$ & $33.1 \pm 5.7$ \\
& & & & & \\
\hline
\end{tabular}

The subjects $(n=6)$ performed bicycle exercise on a constant workload (see Table I) above the lactate threshold under normoxic and hypoxic conditions as described in Methods. The CoA content was determined with a radioenzymatic method in muscle biopsies obtained at rest (preexercise) and after exhaustive exercise. Units are $\mu \mathrm{mol} / \mathrm{kg}$ muscle wet weight. Data are presented as mean $\pm \mathrm{SD}$. No significant differences were detected between hypoxia and normoxia.

$* P<0.05$ exhaustion vs preexercise

$1.89 \pm 0.38 \mathrm{mmol} / \mathrm{kg}$ at rest to $6.30 \pm 2.87 \mathrm{mmol} / \mathrm{kg}$ after exhaustion under normoxic conditions $(P<0.05$, exhaustion vs. rest), and from $1.95 \pm 0.61$ to $8.65 \pm 3.36 \mathrm{mmol} / \mathrm{kg}$ under hypoxic conditions $(P<0.05$, exhaustion vs. rest $)$.

The CoA pool in skeletal muscle is characterized in Table III. In comparison to the preexercise values, exhaustive exercise was associated with an increase in the acetyl-CoA content in all individuals both under normoxic and hypoxic conditions. In contrast, the skeletal muscle content of CoASH, short-chain acyl-CoA, total acid-soluble CoA, long-chain acyl-CoA, and total CoA were not significantly affected by exercise. With exercise, acetyl-CoA expressed as a fraction of the short-chain acyl-CoA content increased from 34-79\% under normoxic conditions, and from 41-69\% under hypoxic conditions. As shown in Fig. 1, in skeletal muscle obtained after exhaustive exercise, the acetyl-CoA/CoASH ratio showed a linear correlation with the lactate concentration, a finding which agrees with the concept that the activity of pyruvate dehydrogenase is regulated by the acetyl-CoA/CoASH ratio (32).

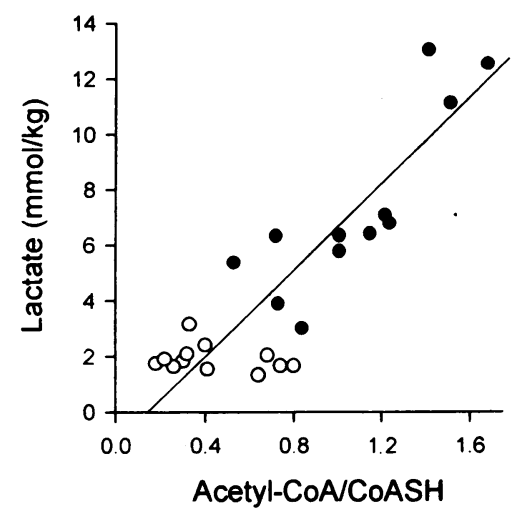

Figure 1. Relationship between the acetyl-CoA/CoASH ratio and the lactate content in skeletal muscle. The acetyl-CoA/CoASH ratio and the lactate content were determined in skeletal muscle of six subjects at rest (open symbols) and after exhaustive exercise (closed symbols) under normoxic and hypoxic conditions as described in Methods. The values obtained under normoxic and hypoxic conditions were not significantly different and are therefore pooled. After exhaustive exercise, a linear correlation was obtained between the two variables $(y=-0.65$ $+7.74 x ; r=0.84, P<0.05)$.

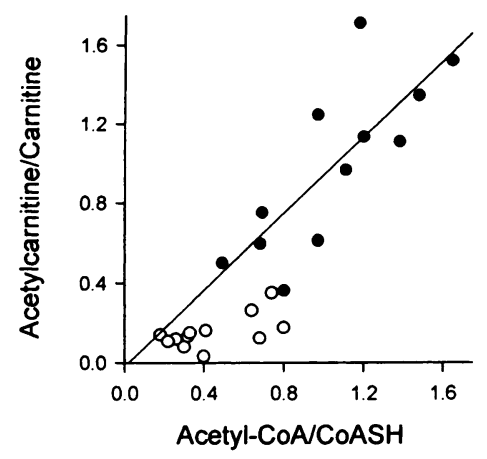

Figure 2. Relationship between the acetyl-CoA/CoASH and the acetylcarnitine/carnitine ratios in skeletal muscle. The acetyl-CoA/CoASH and the acetylcarnitine/carnitine ratios were determined in skeletal muscle of six subjects at rest (open symbols) and after exhaustive exercise (closed symbols) under normoxic and hypoxic conditions as described in Methods. The values obtained under normoxic and hypoxic conditions were not significantly different and are therefore pooled. After exhaustive exercise, a linear correlation was obtained between the two variables $(y=-0.02+0.96 x ; r=0.79, P<0.05)$.

The carnitine pool in skeletal muscle is characterized in Table IV. In agreement with the changes observed in the CoA A pool, exhaustive exercise was associated with a significant increase in the acetylcarnitine content, both under normoxic and hypoxic conditions. However, in contrast to the changes observed in the CoA pool, exhaustive exercise led also to a significant increase in the skeletal muscle short-chain acylcarnitine content and to a corresponding decrease in the free carnitine content. The acetylcarnitine content expressed as a fraction of the short-chain acylcarnitine content decreased from $97 \%$ at rest to $59 \%$ after exhaustive exercise under normoxic conditions, and from 76-72\% under hypoxic conditions. Similar to the CoA pool, the skeletal muscle contents of total acid-soluble carnitine, long-chain acylcarnitine, and total carnitine were not affected by exercise. As shown in Fig. 2, in skeletal muscle obtained after exhaustive exercise, the acetyl-CoA/CoASH and the acetylcarnitine/carnitine ratios showed a linear correlation and were both increased in comparison to the corresponding values at rest.

Table IV. Carnitine Content in Skeletal Muscle

\begin{tabular}{llllll}
\hline & \multicolumn{2}{c}{ Normoxia } & & \multicolumn{2}{c}{ Hypoxia } \\
\cline { 2 - 3 } \cline { 5 - 6 } & Preexercise & Exhaustion & & Preexercise & Exhaustion \\
\hline $\begin{array}{c}\text { Free carnitine } \\
\text { Acetylcarnitine }\end{array}$ & $2.65 \pm 0.64$ & $1.15 \pm 0.52^{*}$ & & $2.70 \pm 0.24$ & $1.34 \pm 0.32^{*}$ \\
$\begin{array}{c}\text { Short-chain } \\
\text { acylcarnitine }\end{array}$ & $0.32 \pm 0.20$ & $0.95 \pm 0.33^{*}$ & & $0.48 \pm 0.19$ & $1.24 \pm 0.24 *$ \\
$\begin{array}{c}\text { Total acid-soluble } \\
\text { carnitine }\end{array}$ & $2.98 \pm 0.69$ & $2.76 \pm 0.56$ & & $3.33 \pm 0.35$ & $3.07 \pm 0.50$ \\
$\begin{array}{c}\text { Long-chain } \\
\text { acylcarnitine }\end{array}$ & $0.14 \pm 0.04$ & $0.15 \pm 0.02$ & & $0.17 \pm 0.03$ & $0.17 \pm 0.03$ \\
Total carnitine & $3.12 \pm 0.72$ & $2.91 \pm 0.57$ & & $3.50 \pm 0.37$ & $3.24 \pm 0.50$
\end{tabular}

The subjects $(n=6)$ performed bicycle exercise on a constant workload (see Table I) above the lactate threshold under normoxic and hypoxic conditions as described in Methods. The carnitine content was determined with a radioenzymatic method in muscle biopsies obtained at rest (preexercise) and after exhaustive exercise. Units are mmolkg muscle wet weight. Data are presented as mean \pm SD. No significant differences were detected between hypoxia and normoxia. $* P<0.05$ exhaustion vs preexerice. 
The relationship between the acetyl-CoA/CoASH and the acetylcarnitine/carnitine pools can be described by the apparent equilibrium constant (apparent $K_{\mathrm{eq}}$ ) of the reaction: CoASH + acetylcarnitine $\rightleftharpoons$ acetyl $-\mathrm{CoA}+$ carnitine, which is mediated by the action of the carnitine acetyltransferase $(10,33)$. As shown in Table V, the apparent $K_{\text {eq }} \mathrm{s}$ were significantly higher at rest, compared to the values obtained after exhaustive exercise. The values of the apparent $K_{\text {eq }}$ s obtained after exercise are close to unity, both under normoxic and hypoxic conditions.

\section{Discussion}

The major findings of the current studies are the maintenance of the skeletal muscle CoASH content after exhaustive exercise, both under normoxic and hypoxic conditions, and the close relationship between the CoA and the carnitine pools in the exercising muscle.

In agreement with results obtained in humans (34), the CoASH content in the resting muscle accounted for $40-50 \%$ of the total acid-soluble CoA content, acetyl-CoA for $\sim 20 \%$, and short-chain acyl-CoAs different from acetyl-CoA (non-acetyl short-chain acyl-CoA equals short-chain acyl-CoA minus acetyl-CoA) for $\sim 30 \%$. After exhaustive exercise, the skeletal muscle content of CoASH, total acid-soluble CoA, and total CoA had not changed significantly, whereas the acetyl-CoA content was increased by a factor of two, both under normoxic and hypoxic conditions. Similar results were obtained in studies characterizing the skeletal muscle $\mathrm{CoA}$ and carnitine pools in the exercising horse (8). These findings indicate that acetyl$\mathrm{CoA}$ is generated at the expense of non-acetyl short-chain acylCoAs in the working skeletal muscle. As discussed below, the $\mathrm{CoASH}$ utilized for the formation of acetyl-CoA is generated predominantly by the reaction of non-acetyl short-chain acylCoAs with carnitine, a reaction mediated by carnitine acyltransferases $(1,11)$. The maintenance of the CoASH content in skeletal muscle during exhaustive exercise therefore appears to be an important consequence of the interaction between the $\mathrm{CoA}$ and the carnitine pools, and the results of the current studies underscore the function of carnitine as a buffer of the CoA pool in the working skeletal muscle $(10,13,14)$. Importantly, the current studies also demonstrate that exhaustion is not caused by depletion of the skeletal muscle CoASH content.

Table V. Relationship between the CoA and the Carnitine Pools in Skeletal Muscle

\begin{tabular}{llc}
\hline & \multicolumn{2}{c}{ Apparent $K_{\mathrm{cq}}$} \\
\cline { 2 - 3 } & Normoxia & Hypoxia \\
\hline Preexercise & $3.74 \pm 1.34$ & $2.57 \pm 1.27$ \\
Exhaustion & $0.95 \pm 0.31^{\ddagger}$ & $0.89 \pm 0.11^{\ddagger}$
\end{tabular}

The subjects $(n=6)$ performed bicycle exercise on a constant workload above the lactate threshold (see Table I) under normoxic and hypoxic conditions as described in Methods. The CoA and the carnitine pools were analyzed in muscle biopsies obtained at rest (preexercise) and after exhaustive exercise. The apparent equilibrium constant (apparent $K_{\text {eq }}{ }^{*}$ ) was calculated for the reaction CoASH + acetylcarnitine $\rightleftharpoons$ acetyl-CoA + carnitine. Data are presented as mean \pm SD. No significant differences were detected between hypoxia and normoxia. $*([$ acetyl-CoA $] \times[$ carnitine $] /([\mathrm{CoASH}] \times[$ acetylcarnitine $]) . \quad{ }^{\ddagger} P<0.05$ exhaustion vs preexercise.
In agreement with earlier studies (3), exercise above the lactate threshold was associated with a three- to fivefold increase in the skeletal muscle short-chain acylcarnitine content and a corresponding decrease in the free carnitine content. These results suggest that, in contrast to the $\mathrm{CoA}$ pool, predominantly free carnitine is used for the formation of short-chain acylcarnitines. Further, the present studies show that acetylcarnitine formation accounted for $50 \%$ of the exercise-associated increase in the skeletal muscle short-chain acylcarnitine content under normoxic conditions, and for $69 \%$ under hypoxic conditions. These results are in agreement with similar studies in humans (5) and in horses (6-8). While acetylcarnitine represents the most abundant acylcarnitine species in the skeletal muscle carnitine pool, both under resting conditions and after exhaustive exercise, it is also interesting to note that the short-chain acylcarnitine content different from acetylcarnitine (non-acetyl short-chain acylcarnitine equals short-chain acylcarnitine minus acetylcarnitine) showed an exercise-associated increase from 0.01-0.66 mmol $/ \mathrm{kg}$ under normoxic conditions, and from $0.15-0.45 \mathrm{mmol} / \mathrm{kg}$ under hypoxic conditions. This finding is consistent with the observation discussed in the preceding paragraph that predominantly non-acetyl short-chain acyl-CoAs are used for the formation of acetyl-CoA. Moreover, it indicates that non-acetyl short-chain acyl-CoAs are converted to the corresponding acylcarnitines and that the $\mathrm{CoASH}$ produced by this reaction is used for the formation of acetyl-CoA. It is well established that non-acetyl short-chain acylcarnitines can be formed from the corresponding acyl-CoAs and can be exported from mitochondria $(12,35)$.

As can be calculated from Table IV, the skeletal muscle short-chain acylcarnitine content increased with exercise by $1.28 \mathrm{mmol} / \mathrm{kg}$ under normoxic conditions, and by $1.10 \mathrm{mmol} /$ $\mathrm{kg}$ under hypoxic conditions. Since these increases are 30-40 times larger than the total skeletal muscle CoA content, they further illustrate the significance of the interaction between the $\mathrm{CoA}$ and the carnitine pools, in order to avoid complete acylation of the functionally important CoASH pool.

As shown in Fig. 2, we found a linear correlation between the acetyl-CoA/CoA and the acetylcarnitine/carnitine ratios in skeletal muscle after exhaustive exercise. Similar correlations have been described in rat hearts (10) or rat hindlimbs (36) perfused with different substrate combinations to modulate the acetylation state of the CoA and the carnitine pools, and in skeletal muscle of exercising horses (8). Interestingly, in agreement with studies in exercising horses (8), the acetylcarnitine/carnitine ratios in skeletal muscle at rest fall below the regression line obtained for the corresponding values after exhaustive exercise. Consequently, the apparent $K_{\text {eq }}$ s describing the reaction between the acetylcarnitine/carnitine and the acetyl-CoA/CoASH pools catalyzed by the carnitine acetyltransferase, were significantly higher at rest, compared to the values obtained after exhaustive exercise (Table V). The apparent $K_{\mathrm{eq}} \mathrm{s}$ obtained after exercise are close to 0.6 , the value of the in vitrodetermined $K_{\text {eq }}$ for this reaction (33). The difference in the apparent $K_{\text {eq }}$ s obtained at rest and after exhaustive exercise may be explained by the compartmentation of the cellular CoA and carnitine pools. The cellular CoA pool in rat hearts is localized to $>90 \%$ intramitochondrially, whereas the carnitine pool is localized to $>90 \%$ extramitochondrially (37). Since the skeletal muscle carnitine pool is two orders of magnitude larger than the CoA pool (8) (Tables III and IV), the intramitochondrial concentration of total $\mathrm{CoA}$ and carnitine are estimated to be in the same range, at $\sim 2 \mathrm{mmol} /$ liter (37). Based on these 
considerations, it can be hypothesized that, under resting conditions, only the mitochondrial CoA and carnitine pools are equilibrated, and that a total cell equilibrium between the two pools is achieved when the flux through the pyruvate dehydrogenase and the generation of acetyl-CoA are increased, as observed in the exercising skeletal muscle.

In agreement with earlier studies $(16,17,38,39)$, exercise under hypoxic conditions was associated with decreased $\mathrm{VO}_{2}$, and increased RER and blood lactate concentrations in comparison to normoxic conditions (Table II). In the current studies, acute hypoxia had no significant effect on the skeletal muscle CoA and carnitine pools, only a trend for an increase in the acetyl- and short-chain acylcarnitine pools can be detected ( Table IV). In comparison, endurance exercise under hypoxic conditions was associated with an increase in the acylcarnitine pool in skeletal muscle (18), suggesting that sustained hypoxia, in particular combined with endurance exercise, could be associated with changes in the skeletal muscle carnitine pool.

In conclusion, the skeletal muscle CoASH content is not significantly altered with exhaustive exercise, whereas the acetyl-CoA and the acetylcarnitine contents both increase and the free carnitine content decreases. The current studies demonstrate the close interaction of the CoA and the carnitine pools in the working skeletal muscle and underscore the importance of the buffer role of carnitine in maintaining the CoASH pool.

\section{Acknowledgments}

The authors thank Dr. R. Billeter, Dr. J. Geiser, and Ms. A. Grossenbacher for technical assistance during the exercise tests, Mrs. B. O'Neill for performing the radioenzymatic assays, and Professor P. J. MeierAbt for reading the manuscript.

The work was financially supported by the Federal Institute of Sport Sciences, Magglingen, Switzerland; by Sigma-Tau, Switzerland; and by grant 32-37280.93 from the Swiss National Science Foundation to $S$. Krähenbühl.

\section{References}

1. Bremer, J. 1983. Carnitine-metabolism and functions. Physiol. Rev. 63:1420-1480.

2. Brass, E. P., and C. L. Hoppel. 1978. Carnitine metabolism in the fasting rat. J. Biol. Chem. 253:2688-2693.

3. Hiatt, W. R., J. G. Regensteiner, E. E. Wolfel, L. Ruff, and E. P. Brass. 1989. Carnitine and acylcarnitine metabolism during exercise in humans. J. Clin. Invest. 84:1167-1173.

4. Lennon, D. L. F., F. W. Stratman, E. Shrago, F. J. Nagle, M. Madden, P. Hanson, and A. L. Carter. 1983. Effects of acute moderate-intensity exercise on carnitine metabolism in men and women. J. Appl. Physiol. 55:489-495.

5. Sahlin, K. 1990. Muscle carnitine metabolism during incremental dynamic exercise in humans. Acta Physiol. Scand. 138:259-262.

6. Foster, C. V. L., and R. C. Harris. 1987. Formation of acetylcarnitine in muscle of horse during high intensity exercise. Eur. J. Appl. Physiol. Occup. Physiol. 56:639-642.

7. Harris, R. C., and C. V. L. Foster. 1990. Changes in muscle free carnitine and in acetylcarnitine with increasing work intensity in the thoroughbred horse. Eur. J. Appl. Physiol. Occup. Physiol. 60:81-85.

8. Carlin, J. I., R. C. Harris, G. Cederblad, D. Constantin-Teodosiu, D. H. Snow, and E. Hultman. 1990. Association between muscle acetyl-CoA and acetylcarnitine levels in the exercising horse. J. Appl. Physiol. 69:42-45.

9. Harris, R. C., C. V. L. Foster, and E. Hultman. 1987. Acetylcarnitine formation during intense muscular contraction in humans. J. Appl. Physiol. 63:440-442.
10. Pearson, D. J., and P. K. Tubbs. 1967. Carnitine and derivatives in rat tissues. Biochem. J. 105:953-963.

11. Ramsay, R. R., and A. Arduini. 1993. The carnitine acyltransferases and their role in modulating acyl-CoA pools. Arch. Biochem. Biophys. 302:307-314.

12. Lysiak, W., K. Lilly, F. DiLisa, P. P. Toth, and L. L. Bieber. 1988. Quantitation of the effect of $\mathrm{L}$-carnitine on the levels of acid-soluble short-chain acyl-CoA and CoASH in rat heart and liver mitochondria. J. Biol. Chem. 263:1151-1156.

13. Brass, E. P., and C. L. Hoppel. 1980. Relationship between acid-soluble carnitine and coenzyme A pools in vivo. Biochem. J. 190:495-504.

14. Bieber, L. L., R. Emaus, K. Valkner, and S. Farrell. 1982. Possible functions of short-chain and medium-chain carnitine acyltransferases. Fed. Proc. 41:2858-2862.

15. Uziel, G., B. Garavaglia, and S. Di Donato. 1988. Carnitine stimulation of pyruvate dehydrogenase complex (PDHC) in isolated human skeletal muscle mitochondria. Muscle \& Nerve. 11:720-724.

16. Cooper, D. M., D. H. Wassermann, M. Vranic, and K. Wasserman. 1986. Glucose turnover in response to exercise during high- and low- $\mathrm{FI}_{02}$ breathing in man. Am. J. Physiol. 251:E209-E214.

17. Jones, N. L., D. G. Robertson, J. W. Kane, and A. Hart. 1972. Effect of hypoxia on free fatty acid metabolism during exercise. J. Appl. Physiol. 33:733738.

18. Decombaz, J., B. Gmuender, G. Sierro, and P. Cerretelli. 1992. Muscle carnitine after strenuous endurance exercise. J. Appl. Physiol. 72:423-427.

19. Carter, A. L., D. L. F. Lennon, and F. W. Stratman. 1981. Increased acetyl carnitine in rat skeletal muscle as a result of high-intensity short-duration exercise. FEBS (Fed. Eur. Biochem. Soc.) Lett. 126:21-24.

20. Spurway, N. C. 1992. Aerobic exercise, anaerobic exercise and the lactate threshold. Br. Med. Bull. 48:569-591.

21. Bergström, J. 1975. Percutaneous needle biopsy of skeletal muscle for physiological and clinical research. Scand. J. Clin. Lab. Invest. 35:609-616.

22. Ferretti, G., B. Kayser, F. Schena, D. L. Turner, and H. Hoppeler. 1992. Regulation of perfusive $\mathrm{O}_{2}$-transport during exercise in humans: effects of changes in haemoglobin concentration. J. Physiol. (Camb.) 455:679-688.

23. Cederblad, G., and S. Lindstedt. 1972. A method for the determination of carnitine in the picomole range. Clin. Chim. Acta. 37:235-243.

24. Cederblad, G., J. I. Carlin, D. Constantin-Teodosiu, P. Harper, and E. Hultman. 1990. Radioisotopic assays of CoASH and carnitine and their acetylated forms in human skeletal muscle. Anal. Biochem. 185:274-278.

25. Pande, S. V., and M. N. Caramancion. 1981. A simple radioisotopic assay of acetylcarnitine and acetyl-CoA at picomolar levels. Anal. Biochem. 112:3038.

26. Krähenbühl, S., and E. P. Brass. 1991. Inhibition of propionyl-CoA synthetase activity by organic acids. Reversal of propionate inhibition of pyruvate metabolism. Biochem. Pharmacol. 41:1015-1023.

27. Michal, G., and H. U. Bergmeyer. 1987. Coenzyme A and derivatives. In Methods of Enzymatic Analysis, Volume VII. H. U. Bergmeyer, J. Bergmeyer, and M. Grassl, editors. Verlag Chemie, Weinheim, Germany. 165-169.

28. Stadtman, E. R. 1957. Preparation and assay of acylcoenzyme A and othe thiol esters; use of hydroxylamine. Methods Enzymol. 3:931-941.

29. Lowry, O. H., N. J. Rosebrough, A. L. Farr, and R. J. Randall. 1951. Protein measurement with the Folin phenol reagent. J. Biol. Chem. 193:265-275.

30. Lilienthal, J. L., K. L. Zierler, B. P. Folk, R. Buka, and M. J. Riley. 1950. A reference base and system for analysis of muscle constituents. J. Biol. Chem. 182:501-508

31. Armitage, P., and G. Berry. 1987. Statistical Methods in Medical Research Blackwell Scientific Publications, Oxford. 214-263.

32. Bremer, J. 1969. Pyruvate dehydrogenase, substrate specificity and product inhibition. Eur. J. Biochem. 8:535-540.

33. Fritz, I. B., S. K. Schultz, and P. A. Srere. 1963. Properties of partially purified carnitine acetyltransferase. J. Biol. Chem. 238:2509-2517.

34. Carroll, J. E., M. H. Brooke, A. Villadiego, B. J. Norris, and J. I. Trefz

1986. 'Dystrophic' lipid myopathy in two sisters. Arch. Neurol. 43:128-131.

35. Solberg, H. E., and J. Bremer. 1970. Formation of branched chain acylcarnitines in mitochondria. Biochim. Biophys. Acta. 222:372-380.

36. Spriet, L. L., D. J. Dyck, G. Cederblad, and E. Hultman. 1992. Effects of fat availability on acetyl-CoA and acetylcarnitine metabolism in rat skeletal muscle. Am. J. Physiol. 263:C653-C659.

37. Idell-Wenger, J. A., L. W. Grotyohann, and J. R. Neely. 1978. Coenzyme A and carnitine distribution in normal and ischemic hearts. J. Biol. Chem. 253:4310-4318

38. Linnarsson, D., J. Karlsson, L. Fagraeus, and B. Saltin. 1974. Muscle metabolites and oxygen deficit with exercise in hypoxia and hyperoxia. J. Appl. Physiol. 36:399-402.

39. Ibanez, J., R. Rama, M. Riera, M. T. Prats, and L. Palacios. 1993. Severe hypoxia decreases oxygen uptake relative to intensity during submaximal graded exercise. Eur. J. Appl. Physiol. Occup. Physiol. 67:7-13. 